\title{
Observations from Australasia using the Gravitational Microlensing Technique
}

\author{
Philip Yock \\ Faculty of Science, University of Auckland, Auckland, New Zealand \\ p.yock@auckland.ac.nz
}

\begin{abstract}
The astronomical technique of gravitational microlensing provides new opportunities to make measurements which are difficult or impossible by other methods, or which are complementary to those obtained more directly. These include detection of dark matter, determination of galactic structure, measurement of limb darkening of stars, and searches for extra-solar planets. The technique is best suited to the southern sky, and several observations have been made from Australasia. A sample of these observations is described here. A case is also made for a telescope at the Antarctic dedicated to gravitational microlensing.
\end{abstract}

Keywords: galaxies: halos — planetary systems — stars: atmospheres — techniques: miscellaneous and spectroscopic

\section{Introduction}

The new technique of gravitational microlensing has developed rapidly since its introduction to astronomy in 1993. During that year the MACHO group in Australia, and the EROS and OGLE groups in Chile, made the first observations (Alcock et al. 1993; Aubourg et al. 1993; Udalski et al. 1993). These were quickly followed by observations by the DUO group (Alard, Mao \& Guibert 1995). All these groups observed the apparent brightening of a distant star caused by the lens-like effect of a closer, collinear star, as had been predicted by Einstein (1936). The brightening was found to follow the characteristic achromatic light curve calculated by Paczynski (1986) as the stars moved into, and out of, collinearity. Paczynski had proposed using the technique to search for brown dwarfs in the dark halo of the Galaxy through their lensing effect on stars in the Magellanic Clouds. Subsequently, other uses for the technique were proposed, including studies of galactic structure, stellar spectra, stellar atmospheres and extra-solar planets. Other groups were formed to exploit these applications, some with telescopes in Australasia.

In this paper, the main observations that have been carried out from Australasia to date are described. A proposal to include Antarctica as an observing site in the future is also described. For the most part the content follows an invited talk given by the author to the Joint Meeting of the Astronomical Society of Australia and the Royal Astronomical Society of New Zealand that was held in Sydney in July 1999.

The observations from Australasia have been made by groups known as MACHO, GMAN, PLANET, MPS and MOA. All the fields mentioned above have

(C) Astronomical Society of Australia 2000 been studied, viz. dark matter, galactic structure, stellar spectra, stellar atmospheres and extra-solar planets. These are dealt with in turn in this paper. Most of the groups mentioned above maintain web sites on their work. These are at:

MACHO: http://wwwmacho.mcmaster.ca/

PLANET: http://thales.astro.rug.nl/ planet/

MOA: http://www.phys.vuw.ac.nz/scps/moa/

MPS: http://bustard.phys.nd.edu/MPS/

EROS: http://www.lal.in2p3.fr/recherche/eros/

OGLE: http://www.astrouw.edu.pl/ ftp/ogle/

The last two groups, EROS and OGLE, are the Chilean based ones. The field of gravitational lensing as a whole, including its history dating back to 1804 , was comprehensively reviewed recently by Wambsganss at:

http://www.livingreviews.org/Articles/Volume1/1998-12wamb/.

\section{Dark Matter}

The study of dark matter, and in particular brown dwarfs, was the first application of the gravitational microlensing technqiue to be proposed, and it motivated the early observations by the pioneering groups in the field, viz. MACHO, EROS and OGLE. The technique is particularly suited to the study of brown dwarfs or similar dark objects because it does not rely on optical detection.

Paczynski (1986) showed that, if the Galactic halo is composed of brown dwarfs, then approximately one star in a million in the Magellanic Clouds would be lensed at any one time by a brown dwarf for a duration of $\sim 140 \sqrt{M / M_{\odot}}$ days. Here $M$ denotes the mass of the brown dwarf, which is $<0 \cdot 08 M_{\odot}$. Hence the expected duration for these lensing events is $\sim 40$ days or less. The MACHO group has now monitored several million stars in the Magellanic 


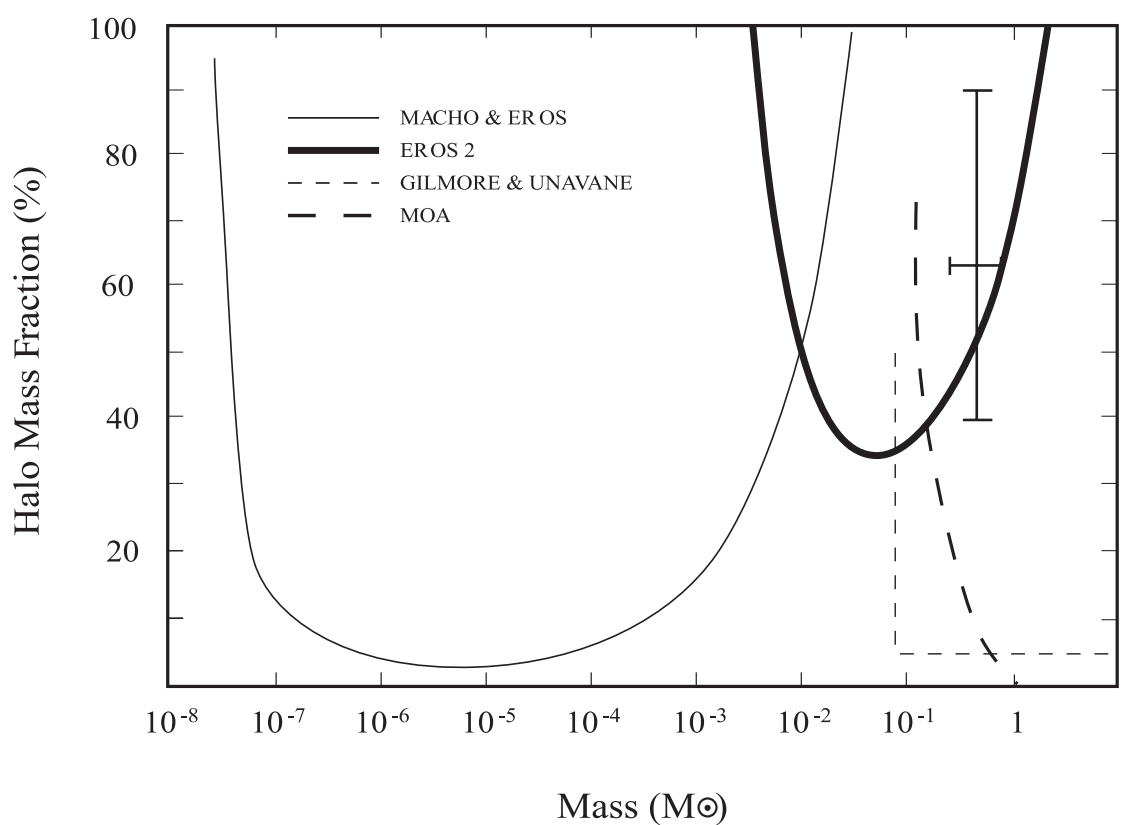

Figure 1-Upper limits to mass fractions of halo objects of various masses determined by Alcock et al. (1998), thin line; by Afonso et al. (1999), thick line; by Gilmore \& Unavane (1998), thin dashed line; and by Abe et al. (1999), thick dashed line. The possible detection is by Alcock et al. (1997a). The limits by Gilmore \& Unavane, and by Abe et al., were obtained by surface photometry of external galaxies. They apply to main-sequence stars only. Note that, at a recent meeting (Microlensing 2000, Cape Town), the EROS group reported a lower limit for halo objects of stellar mass than that shown above, and the MACHO group reported a lower percentage for their possible detection.

Clouds for several years. Amongst their database of LMC stars to 1997, no events were found with duration less than 20 days. A similar result was obtained by the EROS group working from Chile. These results were combined to yield a $95 \%$ confidence upper limit of $\sim 10 \%$ on the halo-mass-fraction of low-mass brown dwarfs with masses in the range $\left(10^{-7}-10^{-2}\right) M_{\odot}$ (Alcock et al. 1998). This result eliminates low-mass brown dwarfs, planets that have been ejected from planetary systems, and any other compact non-luminous objects in the stated mass range as a significant component of halo dark matter for standard models of the halo.

The MACHO and EROS groups extended their searches to include events of longer duration corresponding to brown dwarfs and other non-luminous objects with masses in the range $(0 \cdot 01-1) M_{\odot}$. The EROS group did not detect any events caused by halo objects in this mass range in the direction of the SMC (Afonso et al. 1999). The MACHO group has, however, recorded several events in this mass range in their database towards the LMC, corresponding to a halo fraction of $\sim 50 \%$ of objects with mass $\sim 0.5 M_{\odot}$ (Alcock et al. 1997a). The result, which is marginally consistent with the above result of the EROS group, was generally unexpected. It has proven difficult to interpret.

The above results are shown collectively in Figure 1. This figure also includes data from Gilmore \& Unavane (1998) and the MOA group (Abe et al. 1999) that were obtained by surface photometry of external galaxies. The surface photometry data apply to main-sequence stars, i.e. to red dwarfs. Similarly restrictive limits on the abundance of red dwarfs in the Galactic halo have been obtained from studies of the Hubble deep field (Graff \& Freese 1996; Flynn, Gould \& Bahcall 1996).

It is clear that the objects being detected by the MACHO group cannot be red dwarfs. Two types of interpretation of the data have been proposed. The first assumes a halo comprised mainly of old white dwarfs (Alcock et al. 1997a) or of primordial black holes (Nakamura 1998). The white dwarf hypothesis requires the initial mass function to be strongly peaked at $\sim 2 M_{\odot}$, to enable the white dwarfs to cool sufficiently to escape detection in other surveys (Chabrier, Segretain \& Mera 1996), and it leaves unexplained the metal enrichment that would be expected to accompany them (Gibson \& Mould 1997). The primordial black hole hypothesis requires their mass function to be peaked at $\sim 0 \cdot 5 M_{\odot}$, surprisingly close to the mass of a normal star. Both these interpretations may be tested in the future by independent observations. Direct observations of white dwarfs should be possible if they are a major component of the halo (Chabrier 1999), and gravitational mergers of primordial black holes may be detectable if primordial black holes are a major component (Nakamura 1998).

The second type of interpretation that has been proposed for the MACHO events has potential implications for galactic structure. Sahu (1994) 
proposed that foreground stars in the LMC may lens background stars in the LMC, a process known as 'self lensing'. Initially, the expected event rate for self-lensing was expected to be too low to account for the observations (Gould 1995a). However, examples of self-lensing were subsequently detected (see Section 5.2 below), and these lend weight to Sahu's hypothesis. Modified models of the LMC have been proposed that may accommodate a high self-lensing rate (Aubourg et al. 1999; Salati et al. 1999; Weinberg 2000), and methods have been proposed to test such models (Zhao 1999a, 1999b, 2000; Graff et al. 2000). In a related proposal, Evans et al. (1998) proposed significant flaring and/or warping of the disk of the Milky Way to account for the MACHO events, a possibility that may also be tested by direct observation.

In summary, the microlensing experiments have shown conclusively that halo dark matter is not composed mainly of brown dwarfs. Not all the data that have been obtained to date have been explained yet. Possibilities have been proposed, including old white dwarfs, primordial black holes, or modifications to current models of galactic structure. These may all be tested through independent observations. Also, further applications of the microlensing technique may yet reveal that brown dwarfs provide a substantial fraction of the mass of the Galactic Bulge.

\section{Galactic Bar}

The OGLE and MACHO groups included the Galactic bulge in their original targets for gravitational microlensing. Both groups reported a significantly higher event rate for microlensing than was expected (Udalski et al. 1994; Alcock et al. 1995). The high rate can be explained by assuming the Galactic bulge is bar shaped (Zhao, Rich \& Spergel 1996). In this model, stars from the near side of the bar lens stars from the far side. Supporting evidence for the model was obtained by the OGLE group who observed a systematic trend in the apparent magnitudes of red clump stars across the bulge (Stanek et al. 1997). These authors found that the microlensing and red clump data can be jointly fitted by a bar inclined at $20^{\circ}-30^{\circ}$ to our line of sight. These results are consistent with earlier hints of a barred structure (e.g. Blitz \& Spergel 1991).

\section{Stellar Spectra}

A beautiful application of gravitational microlensing uses the amplification of the effect to effectively increase the size of a telescope to carry out observations of faint objects that would otherwise be impossible. The feasibility of this application has been ably demonstrated by Lennon et al. (1996, 1997) and by members of the MACHO group (Minniti et al. 1998). Minniti et al. measured the abundance of the rare element lithium in a main sequence bulge star using Keck I when the star was magnified by $\sim 1$ magnitude, thereby effectively converting the diameter of the telescope to $15 \mathrm{~m}$. The microlensing event that was used for this application was MACHO-97-BLG-45, the forty-fifth event found towards the galactic bulge during 1997 by the MACHO group.

This application of gravitational microlensing utilises the achromaticity of the effect, and also the predictability of the time of peak amplification once an event has commenced. The latter feature allows the requisite telescope scheduling to be readily carried out. The above lithium abundance measurement would not have been possible using conventional techniques and existing telescopes. Further such measurements are expected to determine the chemical composition and enrichment history of the bulge.

\section{Stellar Atmospheres}

The study of stellar atmospheres by the gravitational microlensing provides an interesting application of the technique. Two methods are possible. Examples of each are given below.

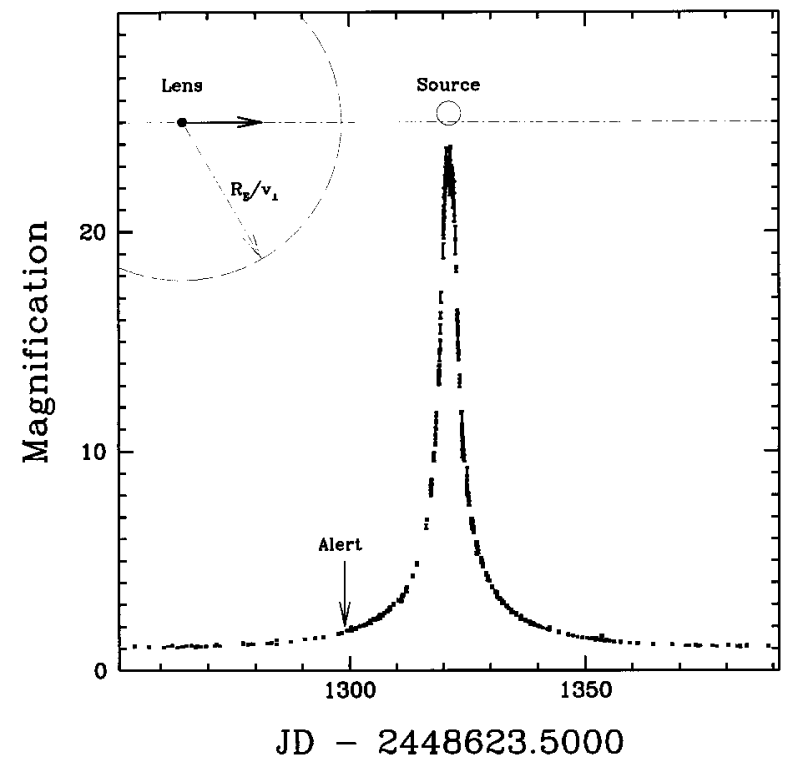

Figure 2-Light curve of gravitational microlensing event MACHO-1995-BLG-30. The additional schematic relates the scale of the lens's Einstein radius to the angular size of the source star, and indicates transit of the lens across the source face. The Einstein radius $R_{E}$ is the impact parameter of the light at the lens plane assuming the lens to be perfectly aligned with the source.

\subsection{MACHO-95-BLG-30}

During July 1995 the MACHO group reported a microlensing event in progress with unusual properties. The star being lensed was a red giant, and the lens trajectory was predicted to transit it. Photometric and spectroscopic observations of the event were requested from several locations, and 


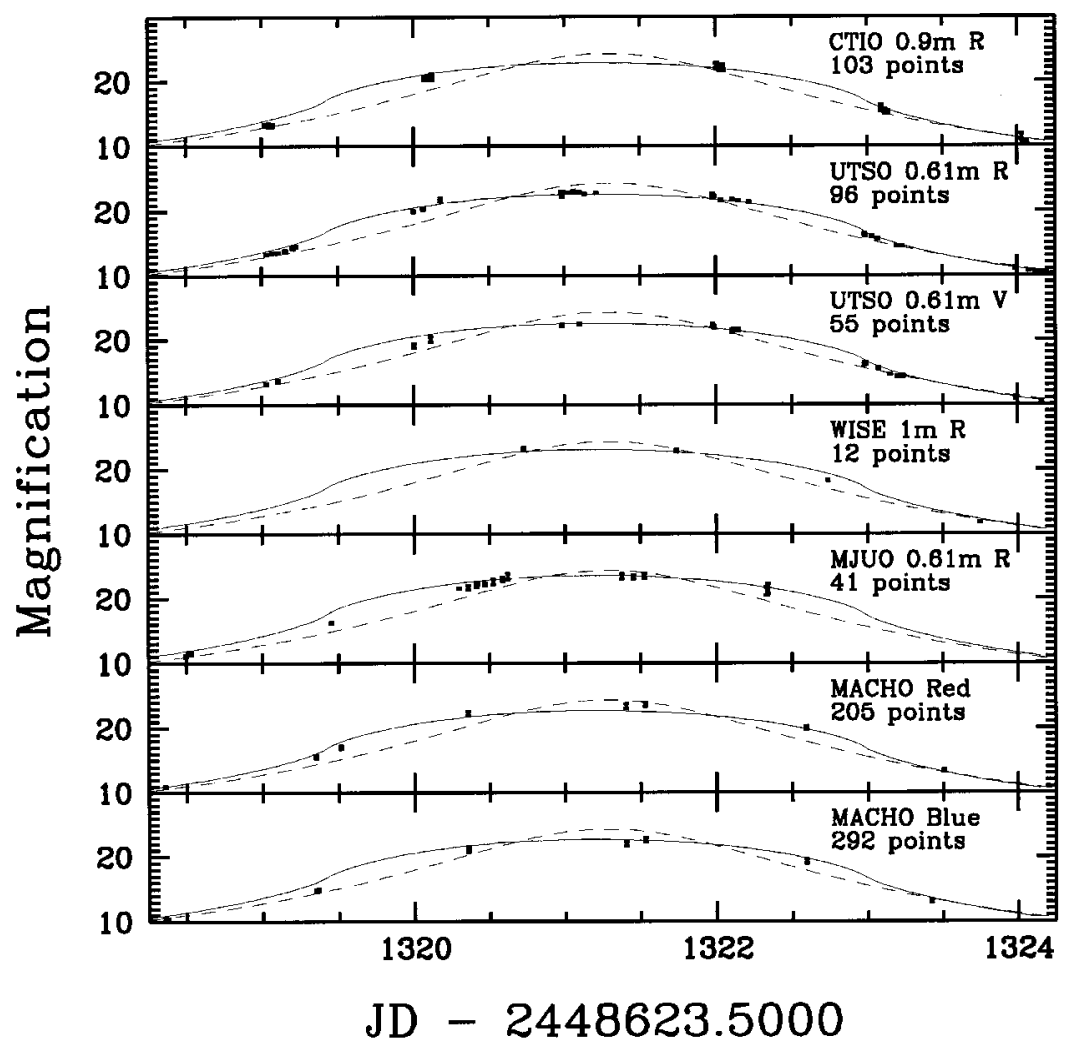

Figure 3-Peak structure of microlensing event MACHO-1995-BLG-30, showing the best standard microlensing fit to the data (dashed curve), and an extended source microlensing fit incorporating source limb-darkening (solid curve).

these were carried out (Alcock et al. 1997b). The light curve for the event is shown in Figures 2 and 3. These include data from Australia, Chile, Israel and New Zealand.

The above data for event MACHO-95-BLG-30 clearly show the effect of the finite size of the source star. As the lens transits the face of the source star, different parts of it are preferentially amplified. Limb darkening and stellar spots on the source star may then be detected (Heyrovsky, Sasselov \& Loeb 1999; Heyrovsky \& Sasselov 2000a). For event MACHO-95-BLG-30 an improved fit to the data was in fact obtained with a limb darkened model of the star. This is shown in Figure 3. Spectra taken during the event, shown in Figure 4, also showed some variation as the lens transited the source star. These are presently being analysed for the purpose of constructing a model atmosphere of the source star in this event (Heyrovsky \& Sasselov 2000b; Heyrovsky 2000).

\subsection{MACHO-98-SMC-1}

This event illustrates the second technique by which stellar atmospheres may be probed using gravitational microlensing. MACHO-98-SMC-1 was monitored by several groups including the PLANET group (Albrow et al. 1999). This group operates the network of telescopes shown in Figure 5. It enables almost continuous surveillance around the clock, weather permitting, of microlensing events. The lens for MACHO-98-SMC-1 was a binary star. Binary lenses can produce light curves that differ markedly from the single peaked structure shown in Figure 2 for a single lens. Figure 6 shows the light curve obtained by the PLANET group for MACHO-98-SMC-1. The asymptotic peaks occur when the source star crosses a 'caustic'. This is a linear region formed by the binary lens where the amplification is formally infinite (Alcock et al. 1999). The shape of the caustic determined by the PLANET group for MACHO-98-SMC-1 is shown in Figure 7.

The formally infinite amplification at a caustic is suppressed by the finite size of the source star. As the caustic sweeps across the face of the source star, different regions are preferentially amplified. This has two consequences. First, the crossing time can be measured and used to constrain the geometry of the event. Second, limb darkening in the source star can be detected. The first of these goals was achieved by the PLANET group for MACHO-98-SMC-1. They found a crossing time of $\sim 4.25 \mathrm{hr}$ for the event, implying the lens was in the SMC (Albrow et al. 1999). The event was an example of the so-called 'self-lensing' process described in Section 2, and the lens was not in the Galactic halo. 


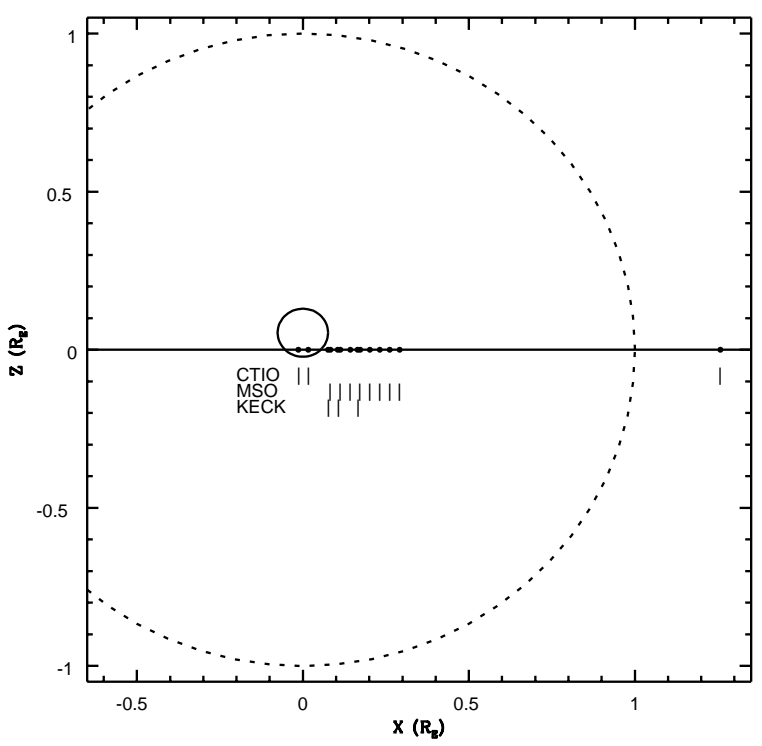

Figure 4-Spectral observations of event MACHO-1995BLG-30 plotted on the source plane. The relative sizes of the star (solid circle) and the lens's Einstein radius (dotted circle) are plotted to scale, in units of $R_{E}$. The solid points show the position of the lens at the times when spectra were taken. The observatories where the spectra were taken are indicated by tick marks.

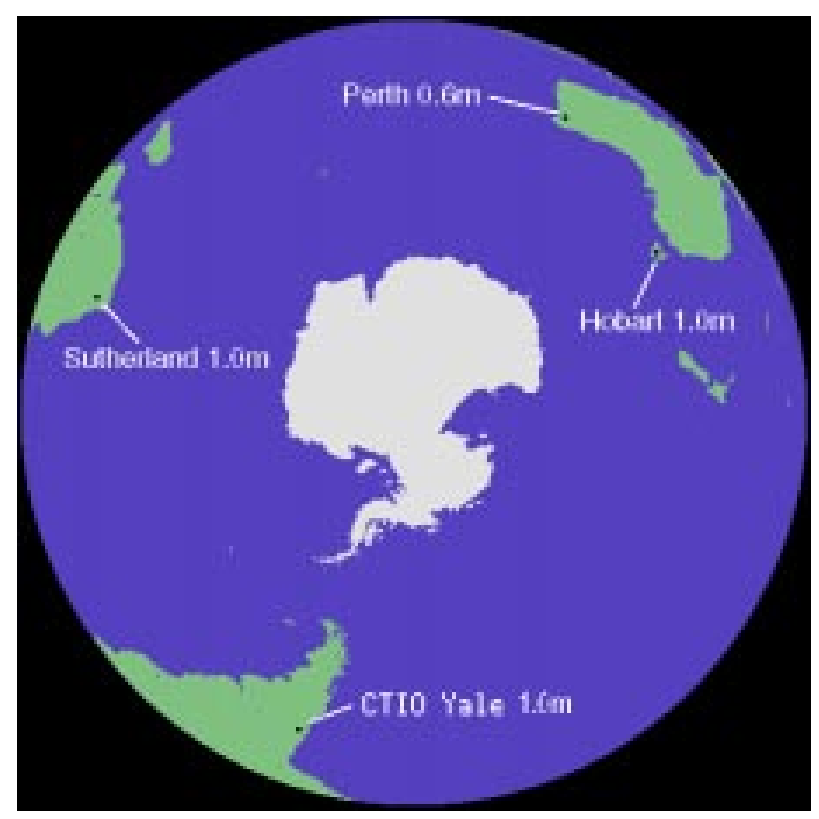

Figure 5-Network of telescopes operated by the PLANET group.

By combining the PLANET data for MACHO-98SMC-1 with data from the EROS, MACHO, GMAN and MPS groups, a detection of limb darkening for the source star in five passbands ranging from I to V was obtained (Afonso et al. 2000). Spectroscopy and photometry of the source star yielded an A6 dwarf classification. Thus, in this instance, limb darkening on a dwarf star some $60 \mathrm{kpc}$ distant was detected! This highlights the extraordinary capability of the microlensing technique. Because the source star for this event was in the SMC, it is likely to be metal-poor. This represents the first measurement of limb darkening of an A type metal-poor star.

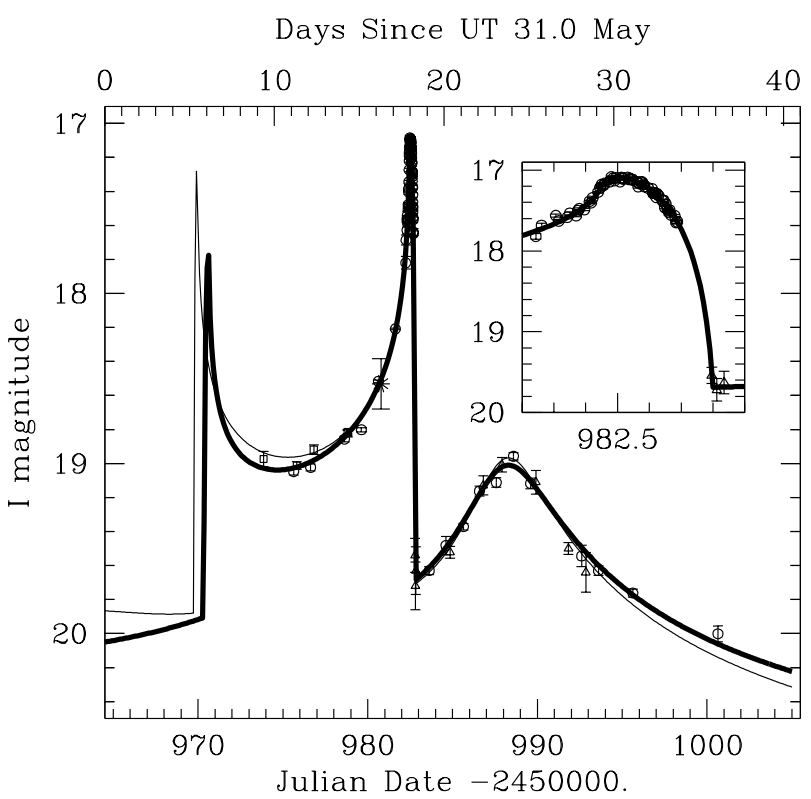

Figure 6-Light curve by the PLANET group for event MACHO-98-SMC-1. The data are from SAAO 1-m (circles), the CTIO 90-cm (squares), the CTIO-Yale 1-m (triangles), and the Canopus 1-m (asterisks) telescopes. The inset covers 0.6 days, corresponding to less than one tick mark on the main figure. The data are binned by day on the main figure. Two fits to the data are shown. These are discussed in Albrow et al. (1999). As is apparent from the figure, the models have similar crossing times.

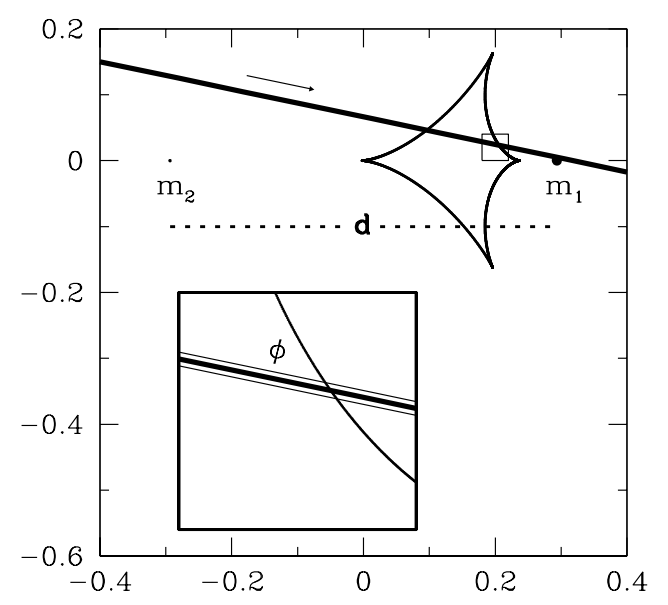

Figure 7-Caustic crossing geometry of event MACHO98-SMC-1 as determined by Albrow et al. (1999). The diamond-shaped curve is the caustic. The thick solid line and the two thinner parallel lines indicate the trajectory and size of the source star. The two components of the binary are shown by circles whose relative sizes are proportional to their masses. The tick marks are in units of the Einstein crossing time $\hat{t}=108 \cdot 4$ days. 


\section{Extra-Solar Planets}

The study of extra-solar planetary systems is one of the more intriguing applications of the gravitational microlensing technique. This application was first proposed by Mao \& Paczynski (1991). Several refinements have since been discussed (Gould \& Loeb 1992; Bennett \& Rhie 1996; Gaudi \& Gould 1997; Wambsganss 1997; Griest \& Safizadeh 1998; Gaudi, Naber \& Sackett 1998; Di Stefano \& Scalzo 1999). The technique may be likened to Rutherford scattering, as shown in Figure 8. Whereas Rutherford used $\alpha$ particles to probe atomic structure, gravitational microlensers use photons to probe planetary structure, by searching for deviations from the light curve produced by a single lens. The technique is promising because, as is indicated in the figure, the region that is probed in microlensing lies a few $\mathrm{AU}$ from the parent star. In contrast, the complementary Doppler technique enjoys greatest sensitivity for planets at orbital radii $<1$ AU (Marcy \& Butler 1998). Applications of the microlensing technique by the PLANET, MOA and MPS groups to events OGLE-98-BLG-14, MACHO-98-BLG-35 and MACHO-97-BLG-41 respectively are described below.

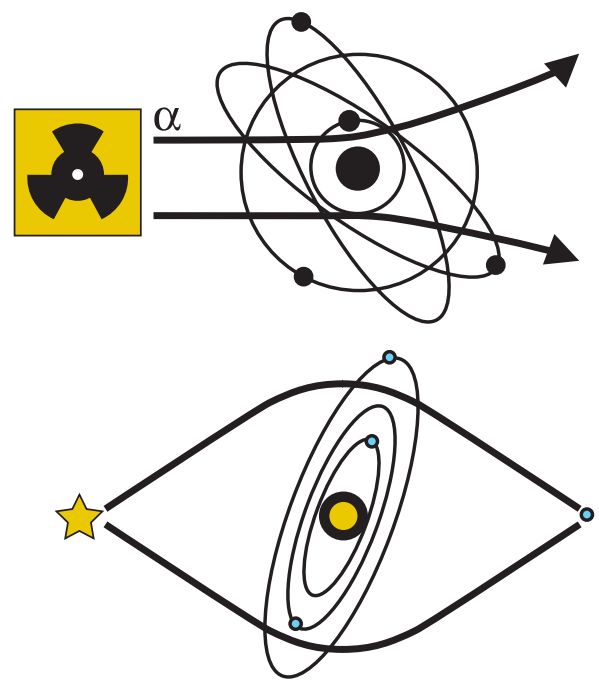

Figure 8-Comparison of Rutherford scattering and gravitational microlensing. The longitudinal scale has been compressed in the lower illustration. Note that the probe is decelerated by electrons in Rutherford scattering, but deflected by planets in gravitational microlensing.

\section{$6.1 O G L E-98-B L G-14$}

The PLANET group made approximately 600 observations of OGLE-98-BLG-14 over a period of $\sim 100$ days (Albrow et al. 2000a). The resulting light curve did not differ appreciably from that of a single lens, even though the estimated detection efficiency for a Jupiter-like planet in the event was $\sim 60 \%$. Thus, although Jupiter analogues could not be ruled out in this event, the detection efficiency was high enough to ensure that future non-detections in similar events would suffice to constrain the abundance of Jupiter-like planets in the galactic bulge. A significant result was obtained for OGLE-98-BLG-14 on the presence of planets heavier than Jupiter. It was found that 'super-Jupiters' with planet-to-star mass ratios of $\epsilon>10^{-2}$ and orbital radii in the range $(0 \cdot 4-2 \cdot 4) R_{E}$ could be excluded with $95 \%$ confidence. Here $R_{E}$ denotes the Einstein radius for the event. ${ }^{1}$

\section{2 $M A C H O-98-B L G-35$}

A significant refinement to the original proposal for planet hunting by Mao \& Paczynski (1991) was made by Griest \& Safizadeh (1998). They found that in microlensing events with high peak amplification, $>20$, a planet always perturbs the light curve near its peak. This happens because the planet produces a small, stellar caustic around the lens, and the source approaches this caustic at the time of peak magnification. The perturbation to the light curve calculated by Griest \& Safizadeh is detectable with high probability for Jupiter-like planets and, depending on the geometry of the event, detectable with finite probability for lighter planets. Because the time of peak magnification of a microlensing event is generally known in advance, the finding by Griest \& Safizadeh appeared to offer a systematic strategy for detecting planets.

An opportunity arose to test the above strategy with event MACHO-98-BLG-35. This reached a peak magnification of $\sim 80$. The peak of the event was monitored by the MPS and MOA groups (Rhie et al. 2000). Their light curves are shown in Figure 9. These include the best fit to the data assuming a lens with and without a planet. The parameters for the best fit with a planet were obtained with a planet mass ratio $\epsilon=7 \times 10^{-5}$ and an orbit radius of $1 \cdot 35 R_{E}$. Assuming a typical value for the lens mass of $\sim 0 \cdot 3 M_{\odot}$, these parameters correspond to a planet with mass between about that of Earth and about twice that of Neptune at an orbit radius of a few AU. The formal significance of the detection is at the $\sim 4.5 \sigma$ level.

Figure 10 shows the same data as Figure 9 but plotted as a ratio to the best fit single lens light curve. Figure 11 shows exclusion regions, at the $6 \cdot 3 \sigma$ confidence level, for planets with various masses. These were calculated by comparing the observed light curves for the event with expected light curves for a dense sampling of possible planetary configurations (Rhie et al. 2000). A solar system analogue is excluded at the $90 \%$ confidence level.

\footnotetext{
1 The Einstein radius $R_{E}$ for bulge events is approximately a few $\mathrm{AU}$, although it is generally not known precisely on an event-by-event basis.
} 
In $88 \%$ of the cases, a Jupiter-like planet would have been detected, and in $19 \%$ of the cases a Saturn-like planet would have been detected. Peale (1997) has proposed the existence of planetary systems in which Jupiter and Saturn are replaced with Neptune-like planets. It is possible that this observation represents the first detection of such a system.

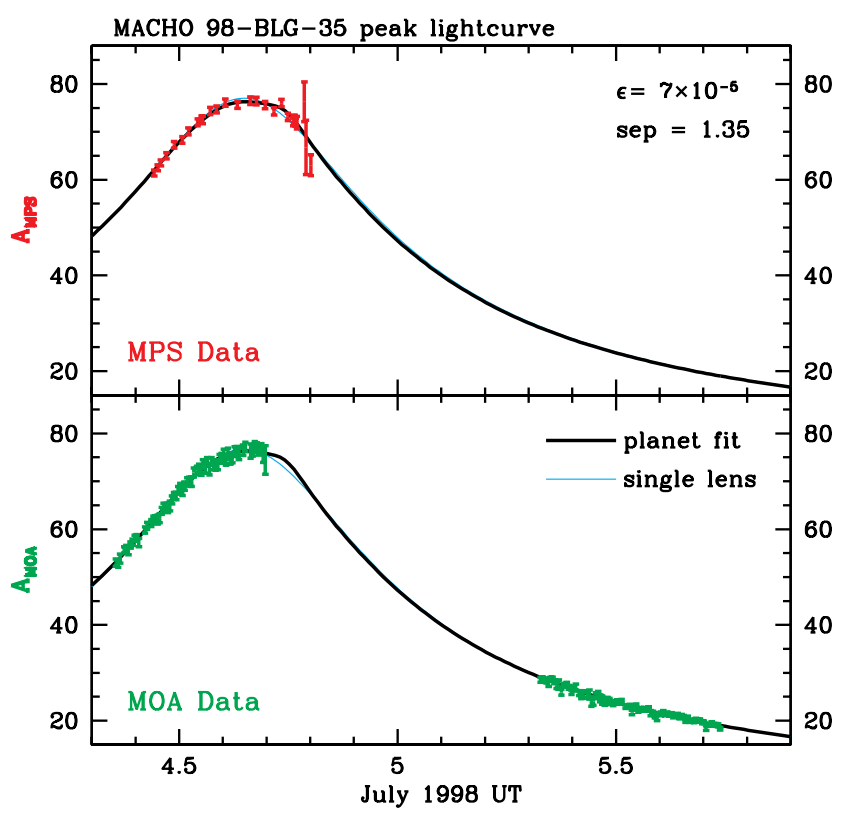

Figure 9-Peak structure of event MACHO-98-BLG-35 as determined by the MPS and MOA groups (Rhie et al. 2000). The error bars are those returned by the DoPHOT point-spread-function fitting routine (Schechter, Mateo \& Saha 1993) with $1 \%$ added in quadrature to allow for possible flat fielding and similar errors that are not included in the DoPHOT routine. The pale curve is the best fit to the data for a lens without a planet, and the heavy curve is the best fit for a lens with a single planet with mass fraction $\epsilon=7 \times 10^{-5}$ and an orbit radius of 1.35 Einstein radii.

The light curves of the MPS and MOA groups for the peak of MACHO-98-BLG-35, shown above in Figures 9 and 10, include measurements made at larger-than-normal air masses, up to an air mass of 3 . The data of both groups were examined for associated deleterious effects. In the case of the MPS data this was explicitly reported in the primary publication (Rhie et al. 2000). In the case of the MOA data, light curves of nearby stars of similar colour, crowdedness and magnitude were examined using the same DoPHOT point-spreadfunction fitting routine that had been used for MACHO-98-BLG-35. It was found that the formal DoPHOT error bars tended to overestimate the statistical errors of check stars, but that a slowly varying systematic error $<1.0 \%$ could have been present. This appeared to be too slowly varying and too small to account for the putative planetary deviation seen at UT $\approx 4.6$ in Figure 10 . It was allowed for by Rhie et al. (2000) by adding $1 \%$ in quadrature to the formal DoPHOT errors.

The PLANET collaboration also observed MACHO98-BLG-35 near its peak (Greenhill 1999). It is hoped that their data will be used to check the above interpretation of the event.

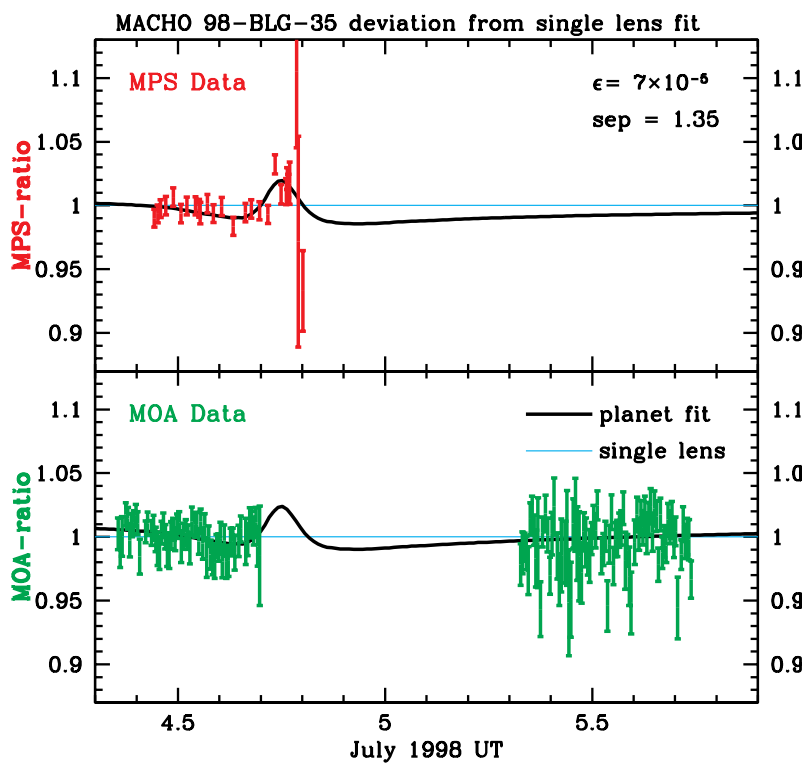

Figure 10 - Same data as in Figure 9 but plotted as a ratio to the best fit to the data for a lens without a planet.

\section{3 $M A C H O-9 \%-B L G-41$}

The microlens event MACHO-97-BLG-41 was monitored by several groups because the light curve showed anomalous behaviour at an early stage, not dissimilar to that expected for a Jupiter-like planet (Glanz 1997). Recently, three analyses of the event have been reported. The MACHO and GMAN groups reported that single-lens and (static) binarylens models could not reproduce their data (Alcock et al. 1999). Subsequently, the MPS group in association with a Wise Observatory team proposed a model of the event in which the lens is assumed to be a planet orbiting a binary star (Bennett et al. 1999). According to this model, the mass ratio of the binary star system is $3 \cdot 8: 1$ and the stars are most likely to be a late $\mathrm{K}$ dwarf and an $\mathrm{M}$ dwarf with a separation of about 1.8 AU. A planet of about 3 Jupiter masses orbits this system at a distance of about 7 AU. Most recently, the PLANET group reported that their dataset, and those of the MACHO, GMAN, MPS and Wise groups, can be accounted for by a rotating binary lens in the Galactic disk with total mass $M \sim 0 \cdot 3 M_{\odot}$ (M-dwarf binary system) and period $P \sim 1 \cdot 5$ yr. (Albrow et al. 2000b). The last proposal does not require the additional complication of a planet, and would seem appealing on the basis of Occam's Razor. Still further data for the event are available that 


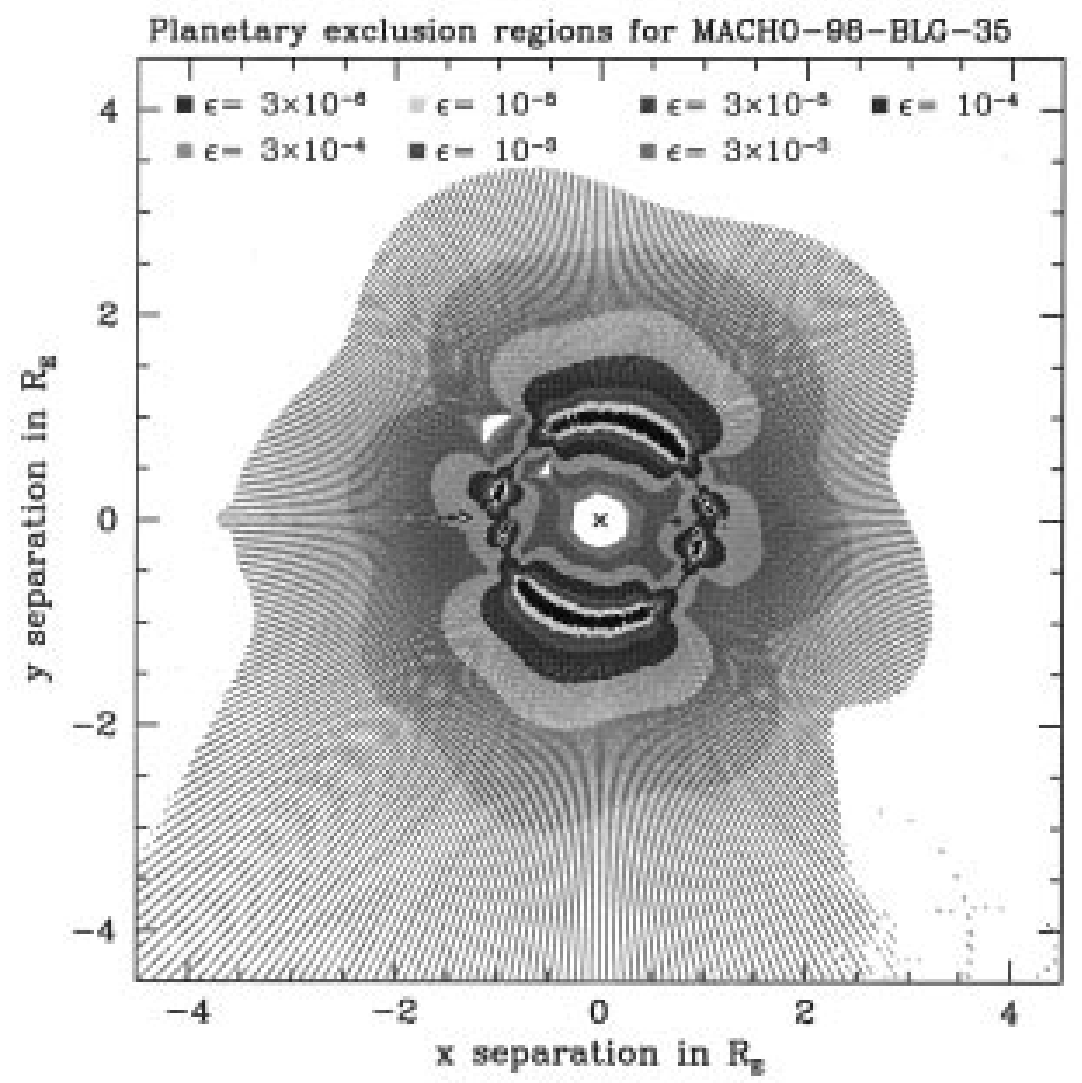

Figure 11-Exclusion regions at the $6 \cdot 3 \sigma$ level of confidence for event MACHO-98-BLG-35 in the lens plane for planets with various mass fractions ranging from Earth-mass $\left(\epsilon=3 \times 10^{-6}\right)$ to three times the mass of Jupiter $\left(\epsilon=3 \times 10^{-3}\right)$. The data are from Rhie et al. (2000). The exclusion region for an Earth-mass planet is the dark, nearly-circular region at the Einstein radius $\left(r=1 R_{E}\right)$. The exclusion regions for heavier planets are successively larger regions surrounding this. The horizontal axis denotes the track of the source star, from right to left.

were not included in the above modeling. It will be interesting to see if a global analysis can pin down the parameters of this interesting event more tightly.

Several hundred observations were made of each of the above three events, OGLE-98-BLG-14, MACHO98-BLG-35 and MACHO-97-BLG-41. For the first and last of these, the crucial observations extended over a period of about 100 days. For the high magnification event, MACHO-98-BLG-35, observations were made over a considerably shorter period without loss of sensitivity. This would appear to confirm the relatively good potential of the high magnification technique of Griest \& Safizadeh (1998).

\section{Gravitational Microlensing from the Antarctic}

To realise the full potential of the gravitational microlensing technique it is necessary to monitor millions of stars with good photometric accuracy at a sampling rate of a few observations per hour in several passbands. The existing network of southern survey and follow-up telescopes (MACHO, OGLE, EROS, GMAN, PLANET, MPS and MOA) do a relatively good job of monitoring the Galactic bulge and the Magellanic Clouds. Further improvement may be expected to occur soon as new image subtraction techniques with better photometric accuracy are refined and incorporated (Alard \& Lupton 1998; Alard 1999).

A quantum leap might be realised with a telescope at the Antarctic. The idea has been raised before (Sahu 1998; Muraki et al. 1999). Such a telescope could monitor southern fields essentially continuously, thus avoiding the not inconsiderable difficulties associated with combining data from different groups using different telescopes and different passbands, and working under different seeing conditions. Losses of data due to inclement weather would also be less serious. To monitor the complete peak of a typical high magnification event from the Antarctic would require good weather for a few days in one location only. Presently, good weather is required simultaneously in Chile, in Australasia and in South Africa (this is like asking for five days without rain for a cricket test in England!). By observing in the infrared, and taking advantage of the exceptionally dry conditions at the Antarctic, one could extend the present measurements to include the centre of the Galaxy. Gould has pointed out this would increase the total event rate (Gould 1995b). A 2-m 
class telescope would be the preferred option to extend the current observations being made with 1-m class instruments.

US and Australian groups have already made considerable progress towards the development of the Antarctic for infrared astronomy (Burton 1996). Observations have been made from the South Pole that confirm its excellent characteristics at infrared wavelengths, and site-testing is in progress at Dome C (see Figure 12) which promises to be even superior (Burton 1998). In view of the above, Dome $\mathrm{C}$ would seem to be a promising site for future development of gravitational microlensing.

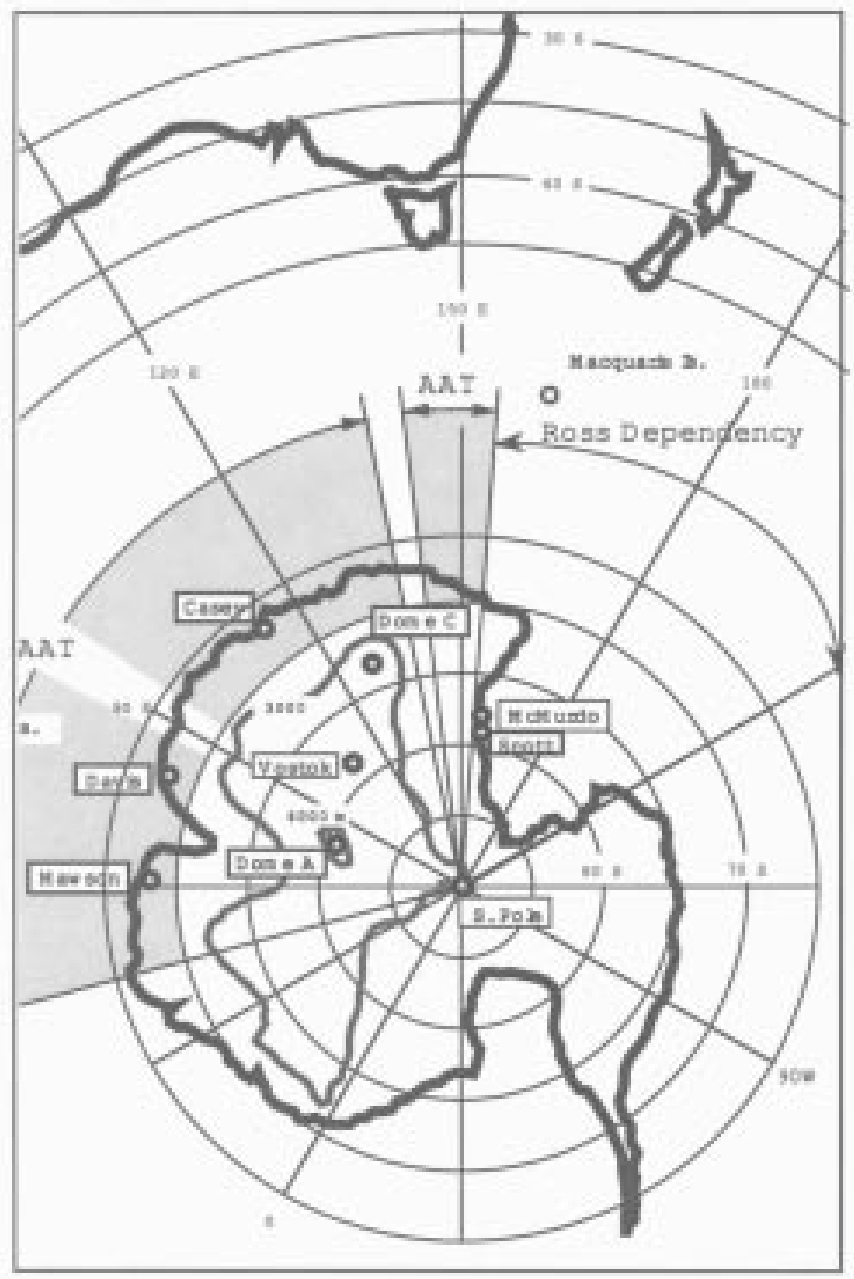

Figure 12-Map of Antarctica from Burton (1996), showing the Plateau bases at the South Pole (USA) and Vostok (Russia) in relation to Australasia. The high Plateau site being considered for a future Antarctic observatory lies inside the 3000-m elevation contour at Dome C. The South Pole is supplied through the US coastal station at McMurdo. The Australian coastal stations at Mawson, Davis and Casey, and the New Zealand Scott Base, are also shown.

\section{Conclusions}

Gravitational microlensing is a useful technique in astronomy. Several new results have been obtained using the technique, including
- elimination of brown dwarfs as halo dark matter

- evidence for a bar in the Galaxy

- spectroscopy of distant faint stars

- measurements of limb darkening on distant stars

- evidence for stars without Jupiter-like planets

- evidence for a low-mass extra-solar planet

Many of these results were made possible by data from the original survey groups, MACHO, OGLE and EROS, being made readily available to follow-up groups. In the future, the Antarctic offers a promising site for extending the technique.

\section{Acknowledgments}

The author thanks Ian Bond, Kem Cook and Nick Rattenbury for commenting on a draft version of the paper, Karen Pollard and Penny Sackett for supplying Figures 5-7, and Michael Burton for Figure 12.

\section{References}

Abe, F., et al. 1999, AJ, 118, 261

Afonso, C., et al. 1999, A\&A, 344, 63

Afonso, C., et al. 2000, ApJ, in press, astro-ph/9907247

Alard, C. 1999, astro-ph/9903111

Alard, C., \& Lupton, R. 1998, ApJ, 503, 325

Alard, C., Mao, S., \& Guibert, J. 1995, A\&A, 300, L17

Albrow, M., et al. 1999, ApJ, 512, 672

Albrow, M., et al. 2000a, ApJ, in press, astro-ph/9909325

Albrow, M., et al. 2000b, ApJ, in press, astro-ph/9910307

Alcock, C., et al. 1993, Nature, 365, 621

Alcock, C., et al. 1995, ApJ, 445, 133

Alcock, C., et al. 1997a, ApJ, 486, 697

Alcock, C., et al. 1997b, ApJ, 491, 436

Alcock, C., et al. 1998, ApJ, 499, L9

Alcock, C., et al. 1999, ApJ, submitted, astro-ph/9907369

Aubourg, E., et al. 1993, Nature, 365, 623

Aubourg, E., et al. 1999, A\&A, 347, 850

Bennett, D., \& Rhie, S. 1996, ApJ, 472, 660

Bennett, D., et al. 1999, Nature, 402, 57

Blitz, L., \& Spergel, D. 1991, ApJ, 379, 631

Burton, M. 1996, PASA, 13, 2

Burton, M. 1998, in Astrophysics from Antarctica, ASP Conf. Series 141, ed. G. Novak \& R. Landsberg (San Francisco: ASP), p. 3

Chabrier, G. 1999, in Third Stromlo Symposium, ASP Conf. Series Vol. 165, ed. B. Gibson et al. (San Francisco: ASP), p. 399

Chabrier, G., Segretain, L., \& Mera, D. 1996, ApJ, 468, L21 Di Stefano, R., \& Scalzo, R. 1999, ApJ, 512, 579

Einstein, A. 1936, Science, 84, 506

Evans, N., et al. 1998, ApJ, 501, L45

Flynn, C., Gould, A., \& Bahcall, J. 1996, ApJ, 466, L55

Gaudi, B., \& Gould, A. 1997, ApJ, 486, 85

Gaudi, B., Naber, R., \& Sackett, P. 1998, ApJ, 502, L33

Gibson, B., \& Mould, J. 1997, ApJ, 482, 98

Gilmore, G., \& Unavane, M. 1998, MNRAS, 301, 813

Glanz, J. 1997, Science, 277, 765

Gould, A. 1995a, ApJ, 441, 77

Gould, A. 1995b, ApJ, 446, L71

Gould, A., \& Loeb, A. 1992, ApJ, 396, 104

Graff, D., \& Freese, K. 1996, ApJ, 456, L49

Graff, D., Gould, A., Suntzeff, N., Schommer, R., \& Hardy,

E. 2000, ApJ, in press, astro-ph/9910360

Greenhill, J. 1999, personal communication

Griest, K., \& Safizadeh, N. 1998, ApJ, 500, 37

Heyrovsky, D. 2000, in preparation 
Heyrovsky, D., \& Sasselov, D. 2000a, ApJ, 529, 69 Heyrovsky, D., \& Sasselov, D. 2000b, in preparation Heyrovsky, D., Sasselov, D., \& Loeb, A. 1999, ApJ, submitted, astro-ph 9902273

Lennon, D., Mao, S., Fuhrmann, K., \& Thomas, G. 1996, ApJ, 471, L23

Lennon, D., et al. 1997, The Messenger, 90, 30

Mao, S., \& Paczynski, B. 1991, ApJ, 374, L37

Marcy, G., \& Butler, R. 1998, ARA\&A, 36, 57

Minniti, D., et al. 1998, ApJ, 499, L175

Muraki, Y., et al. 1999, Prog. Theor. Phys. Suppl., 133, 233

Nakamura, T. 1998, Phys. Reports, 307, 181

Paczynski, B. 1986, ApJ, 304, 1

Peale, S. 1997, Icarus, 127, 269

Rhie, S., et al. 2000, ApJ, in press, astro-ph/9905151
Sahu, K. 1994, Nature, 370, 275

Sahu, K. 1998, in Astrophysics from Antarctica, ASP Conf. Series 141, ed. G. Novak \& R. Landsberg (San Francisco: ASP), p. 179

Salati, P., et al. 1999, A\&A, 350, L57

Schechter, P., Mateo, M., \& Saha, A. 1993, PASP, 105, 1342

Stanek, K., et al. 1997, ApJ, 477, 163

Udalski, A., et al. 1993, Acta Astron., 43, 289

Udalski, A., et al. 1994, Acta Astron., 44, 165

Wambsganss, J. 1997, MNRAS, 284, 172

Weinberg, M. 2000, ApJ, in press, astro-ph/9905305

Zhao, H. 1999a, ApJ, 526, 141

Zhao, H. 1999b, ApJ, 527, 167

Zhao, H. 2000, ApJ, in press, astro-ph/9907191

Zhou, H., Rich, R., \& Spergel, D. 1996, MNRAS, 282, 175 